\title{
Similar chemical structures, dissimilar triplet quantum yields; CASPT2 model rationalizing the trend of triplet quantum yield in nitroaromatic systems
}

\author{
Angelo Giussani ${ }^{*,+\ddagger}$ and Graham A. Worth ${ }^{\dagger}$ \\ ${ }^{\dagger}$ Department of Chemistry, University College London, 20 Gordon Street, London WC1H 0AJ, \\ U.K. \\ *To whom correspondence should be addressed. Email: Angelo.Giussani@uv.es \\ ${ }^{\ddagger}$ Current address: Instituto de Ciencia Molecular, Universitat de València, Apartado22085, ES- \\ 46071 Valencia, Spain
}

\begin{abstract}
The photophysics of nitroaromatics compounds stand out for being characterized by an ultrafast decay into the triplet manifold and by a significant value of the triplet quantum yield. The latter quantity change dramatically depending on the system, as shown for 2-nitronaphthalene, 1nitronaphthalene, and 2-methyl-1-nitronaphthalene, whose triplet quantum yield have been previously measured to be $0.93 \pm 0.15,0.64 \pm 0.12$, and $0.33 \pm 0.05$, respectively (J. Phys. Chem. A 2013, 117, 14100). In this study we rationalize the reported trend for the triplet quantum yield on the basis of the different ability that the excited $S_{1}$ state has to reach a non-previously reported conical intersection with the ground state. Such a path is in competition with the one leading to triplet states, which appears equally favorable in the three systems. The energy barriers from the $S_{1}$ CASPT2//CASSCF minima to a CASPT2 minimum-energy-crossing-point of the $S_{1} / S_{0}$ conical intersection have been computed to follow the same trend as the values of triplet quantum yields in the nitroaromatics system under analysis here. The path has been characterized also for nitrobenzene, obtaining an energy barrier that nicely fits in the derived model, and in agreement with its triplet quantum yield value $(>0.8)$. The ability of the present model to not only rationalize the experimental data of a single molecule but to reproduce a trend for four slightly different systems speaks in favor of its reliability.
\end{abstract}

\section{Introduction}

Nitroaromatic compounds (NCs) are an important class of molecules playing a prominent role in various fields such as atmospheric pollution, energetic materials, and in the drug delivery sector. Being formed as a result of incomplete combustion, ${ }^{1}$ and having mutagenic and carcinogenic properties, NCs such as 1-nitronaphthalene (1NN), 2-nitronaphthalene (2NN), and nitrofluorene constitute a considerable concern for urban air quality. ${ }^{2-5} \mathrm{NCs}$ and in particular nitrobenzene (NB) are common ingredients in energetic explosives, and NB-derived molecules are currently studied in order to obtain energetic compounds with better thermal stability. ${ }^{6}$ Due to their photochemical properties, NB-derived molecules can be used as drugs able to photorelease with precise spatiotemporal resolution nitric oxide (NO), ${ }^{7}$ which in turn is a key molecule having a variety of biological effects in the human body.

Beside their importance in the above mentioned fields, NCs usually display a rich photophysics and photochemistry, making them fascinating systems in the study of molecule-light interactions. Regarding their photochemistry, most NCs photodegradate under UV excitation forming NO and the corresponding aryloxy radical, ${ }^{8-11}$ although other photoproducts, as $\mathrm{NO}_{2}$ and $\mathrm{O}$, can also be obtained. ${ }^{12}$ More intriguingly is the fact that more than one single mechanism can be involved in the formation of the same photoproduct, as is the case of NO photoproduction in NB where so-called roaming mechanisms can operate. ${ }^{13}$ Regarding their photophysics, it is mainly characterized by a rapid decay into the triplet manifold after light absorption. In particular $1 \mathrm{NN}$ is the organic compound with the fastest multiplicity change ever measured, decaying into the triplet state in 
around 100 fs. ${ }^{14-17}$ The same time for triplet state population has also been claimed to characterize $2 \mathrm{NN}$, although recent semiclassical non-adiabatic dynamics concluded that such a time scale is instead due to an $S_{2}-S_{1}$ internal conversion, while the intersystem crossing process leading to the triplet manifold should happen in around $0.7 \mathrm{ps}^{18}$

In order to understand the photophysics of NCs, a great deal of insight can be obtained by the study of a common property in a series of slightly different systems belonging to the same class of compounds. Such an approach was used by Vogt and Crespo-Hernández who experimentally determined, among others properties, the triplet quantum yield for the three molecules 2NN, 1NN and 2-methyl-1-nitronaphthalene $(2 \mathrm{M} 1 \mathrm{NN}) \cdot{ }^{19,20}$ The results from this series of molecules is particularly interesting as, despite their relative chemical similarity, the corresponding triplet quantum yield varies significantly: $0.93 \pm 0.15,0.64 \pm 0.12$, and $0.33 \pm 0.05$ for $2 \mathrm{NN}, 1 \mathrm{NN}$, and 2M1NN, respectively. Performing DFT optimizations and vertical TD-DFT computations, Vogt and Crespo-Hernández obtained the following two results. First, in the ground state at larger torsion angles of the nitro group with respect to the plane of the naphthalene ring, a larger energy gap between the $S_{1}$ and triplet states is observed. Second, the value of the torsion angle in the ground state minima increases along the series, being for example in acetonitrile equal to $0.3,39.3$, and 55.4 degrees for, $2 \mathrm{NN}, 1 \mathrm{NN}$, and $2 \mathrm{M} 1 \mathrm{NN}$, respectively. These results made the authors conclude that the difference in the triplet quantum yield is related to the different torsion angles of the corresponding ground state minima, which in turn strongly influences the energy gap between singlet and triplet states, and then the probability of intersystem crossing.<smiles>Cc1ccc2ccccc2c1[N+](=O)[O-]</smiles>

Figure 1. On the left, atom labeling for $1 \mathrm{NN}$ and $2 \mathrm{M} 1 \mathrm{NN}$; on the right, atom labeling for $2 \mathrm{NN}$.

In the present contribution the reason behind the experimentally recorded trend for the triplet quantum yield in the series 2NN, 1NN, and 2M1NN (see Figure 1) is reconsidered performing abinitio CASPT2//CASSCF and CASPT2//CASPT2 computations. The work has been conducted in the light of the very low fluorescence emission recorded in the systems, ${ }^{14}$ which consequently points to the presence of an accessible non-radiative relaxation process, and of a recently characterized $\mathrm{S}_{1} / \mathrm{S}_{0}$ conical intersection (CI) in the related molecule $\mathrm{NB} .{ }^{21}$ The obtained results shown that CIs displaying similar structural deformations are indeed present also for the three nitronaphthelene systems here under consideration, and that the different ability that the three molecules have to reach such a crossing point constitutes a model able to rationalize the experimental trend of the triplet quantum yield along the 2NN, $1 \mathrm{NN}$, and $2 \mathrm{M} 1 \mathrm{NN}$ series, rather than a different ability to reach a singlet-triplet crossing region leading to the triplet states .

\section{Methods}

The present study has been performed employing the well-tested CASPT2 and CASSCF methods ${ }^{22-}$ ${ }^{24}$ as implemented in the OpenMolcas software. ${ }^{25}$. Geometries optimizations have been performed either at the CASSCF level or at the CASPT2 level, in the latter case computing the required gradients numerically. In both cases, the final energies have been computed at the CASPT2 level to take into account the dynamic correlation effects. No restrictions to the symmetry of the molecule have been imposed ( $C_{1}$ symmetry). The basis set of atomic natural orbital (ANO) of L-type contracted to C,N [4s,3p,1d]/H[2s1p] has been employed. ${ }^{26,27}$ 
An active space composed of 18 active electrons distributed in 14 active orbitals has been used in all the final vertical calculations, and in both CASSCF and CASPT2 optimizations. As shown in Figure S1 of the Supporting Information (SI), the selected active space takes into account both the $\pi$ nature of the system and the lone pairs of the oxygen atoms. Such an active space will be the employed one throughout the work, unless otherwise specified.

Within the CASPT2 calculations, an imaginary level-shift correction of 0.2 au has been used to minimize the effects of possible intruder states. The CASPT2 standard zeroth-order Hamiltonian has been used as originally implemented. ${ }^{23}$ The core orbitals have been frozen in the CASPT2 calculations. Such a CASPT2 approach has been validated in many different studies on organic molecules, providing a correct prediction, description, and interpretation of photophysical experimental data. ${ }^{24,28,29}$

In order to connect some important regions of the potential energy hypersurface (PEH), linear interpolation of internal coordinate (LIIC) calculations have been performed. ${ }^{30}$ Minimum energy crossing points have been optimized using the projected constrained optimization method as recently implemented in Molcas. ${ }^{31}$ The CI optimizations has been performed the CASPT2 level, and not as CASPT2 vertical calculations performed along a CASSCF CI optimization. ${ }^{32}$ In the regions of the potential energy hypersurfaces where two or more states of different spin multiplicity are degenerate, the spin orbit coupling (SOC) has been calculated as described elsewhere. ${ }^{33}$ The Cholesky decomposition has been used to speed up the calculation of two-electron integrals. ${ }^{34}$

In the present work minimum geometries have been characterized for the $S_{0}$ and $S_{1}$ states. For $S_{0}$ the minimizations have been performed at the CASPT2 level, while for $\mathrm{S}_{1}$ at the CASSCF level. In both case, the nature of the obtained minima have been verified computing CASSCF frequencies. As described in the result section, for all minima the computed CASSCF frequencies are all positive, so, even the $\mathrm{S}_{0}$ minima obtained performing CASPT2 optimizations, are indeed CASSCF minima. Consequently, the here adopted approach can be framed in the CASPT2//CASSCF protocol: CASSCF minima, CASPT2 conical intersections, all energies corrected at the CASPT2 level.

\section{Results and discussions}

The significant diversity in the value of the triplet quantum yield in the three molecules $2 \mathrm{NN}, 1 \mathrm{NN}$, and 2M1NN (significantly decreasing along the series), reflects the different ability that the three systems have to decay into the triplet manifold. This in turn can be originated from either the fact that the decay path leading into the triplet manifold is less favorable along the series of molecules, and/or by the presence of a competing decay path not involving the population of the triplet states which becomes more and more favorable along the series of molecules. In order to evaluate the ability of the three systems to decay into the triplet manifold, the main decay path open to the excited $S_{1}$ bright state has been characterized from the respective ground-state minima. The latter equilibrium geometries have been obtained at the CASPT2 level, since for the related system NB CASSCF-optimized ground state structures have been shown to display geometrical parameters that differ significantly with respect to the ones resulting from electron diffraction measurements, especially regarding the NO bond distances. ${ }^{21-23}$ In the CASPT2 optimizations a CAS $(18,14)$ active space has been employed, only the ground state root has been computed, and the corresponding CASPT2 gradients have been evaluated numerically using the OpenMolcas software.

Due most probably to the use of just one root, the CAS $(18,14)$ active space differ from the one later used for the computation of excited states by the presence of two sigma orbitals instead that the full valence $\pi$ space. The same effect was observed in a previous study on NB performed with a similar computational protocol. ${ }^{21}$ In order to characterize the $\mathrm{S}_{0}$ minima so obtained, $\operatorname{CASSCF}(18,14)$ 
frequencies computations were performed, which have provided for all three cases no imaginary frequencies. CASPT2 frequencies computations have not been attempted due to the high computational cost required for the systems here under analysis, and for the limited precision that it is expected out of a numerical evaluation of them, so the frequencies at the computed $\mathrm{S}_{0}$ minima have been evaluated at the CASSCF level. We can however conclude, based on the obtained CASSCF $(18,14)$ frequencies, that the CASPT2 ground-state minima are at least true CASSCF minima. The so obtained $\mathrm{S}_{0}$ minima are hereafter label as ${ }^{1}$ (gs) $\min$.

As shown in Figure 2, the three ${ }^{1}$ (gs) min minima display similar geometrical parameters, the most remarkable difference being the angle formed between the plane of the rings and the nitro group, as reflected by the values of the $\mathrm{C}_{10} \mathrm{C}_{1} \mathrm{~N}_{11} \mathrm{O}_{12}$ and $\mathrm{C}_{2} \mathrm{C}_{1} \mathrm{~N}_{11} \mathrm{O}_{12}\left(\mathrm{C}_{1} \mathrm{C}_{2} \mathrm{~N}_{11} \mathrm{O}_{12}\right.$ and $\mathrm{C}_{3} \mathrm{C}_{2} \mathrm{~N}_{11} \mathrm{O}_{12}$ for 2NN) dihedral angles. In qualitative agreement with previous DFT computations, ${ }^{19}$ the ground state minimum is a planar geometry for the $2 \mathrm{NN}$ system, while for both $1 \mathrm{NN}$ and $2 \mathrm{M} 1 \mathrm{NN}$ the nitro group is out of the plane defined by the rings by around 45 and 70 degrees, respectively. Comparing the CASPT2 $(18,14) 1 \mathrm{NN} \mathrm{S}_{0}$ minimum with a previously CASSCF $(16,13)$ optimized structure ${ }^{37}$ (see Figure S2), the most significant differences are seen in the values of the NO bond lengths, between 0.02-0.04 Å shorter at the CASSCF level.
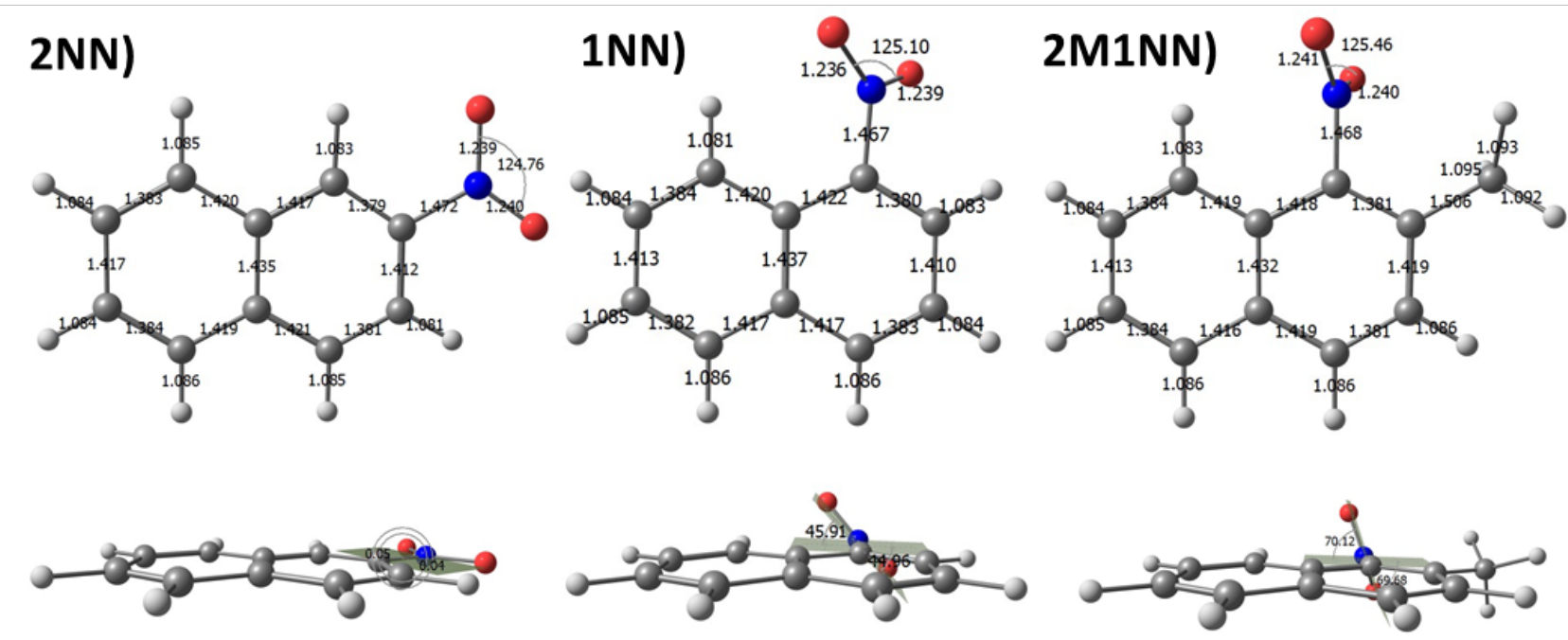

Figure 2. ${ }^{1}$ (gs) $)_{\min }$ minima of $2 \mathrm{NN}, 1 \mathrm{NN}$, and $2 \mathrm{M} 1 \mathrm{NN}$. Selected bond lengths (in $\AA$ ), angles, and dihedral angles (in degrees) are also reported.

The electronic structure of the obtained minima have been characterized computing the low-lying singlet and triplet excited states. In particular four singlet and five triplet excited states have been calculated, and the corresponding vertical energies, natures, and oscillator strengths are reported in Table 1. In all cases a very similar ordering of the states is observed, and the $\mathrm{S}_{1}{ }^{1}\left(\mathrm{n}_{\mathrm{A}} \pi^{*}\right)$ state appears nearly degenerate with the $\mathrm{T}_{3}{ }^{3}\left(\pi_{\mathrm{O}} \pi^{*}\right)$ state. The latter two states are strongly coupled, as reflected by the corresponding SOC terms, equal to $75.71,76.15$, and $78.26 \mathrm{~cm}^{-1}$ for $2 \mathrm{NN}, 1 \mathrm{NN}$, and $2 \mathrm{M} 1 \mathrm{NN}$, respectively. The second lowest singlet state, $\mathrm{S}_{2}$, having a ${ }^{1}\left(\mathrm{~L}_{b} \pi \pi^{*}\right)$ nature, is separated from the $S_{1}{ }^{1}\left(n_{A} \pi^{*}\right)$ state in the three molecules by $0.20,0.34$, and $0.28 \mathrm{eV}$, respectively. The nature of the characterized states is exemplified for 2NN in Table S1.

In the recording of the triplet quantum yields, Vogt and Crespo-Hernández employed an excitation wavelength of $355 \mathrm{~nm}$ (3.49 eV), accordingly to which they claimed to initially populate the $\mathrm{S}_{1}$ state. ${ }^{19}$ From TD-DFT computations, Vogt and Crespo-Hernández concluded that the lowest and initially excited singlet state has $\pi \pi^{*}$ nature. On the basis of our results, however, it is concluded that at such experimental excitation the $\mathrm{S}_{1}{ }^{1}\left(\mathrm{n}_{\mathrm{A}} \pi^{*}\right)$ state is populated, being the only state below such an excitation energy apart from the $S_{2}{ }^{1}\left(\mathrm{~L}_{\mathrm{b}} \pi \pi^{*}\right)$ state in $2 \mathrm{NN}$, which is however expected to 
decay in an ultrafast fashion into the ${ }^{1}\left(\mathrm{n}_{\mathrm{A}} \pi^{*}\right)$ state. ${ }^{18}$ A rapid decay from the ${ }^{1}\left(\mathrm{~L}_{\mathrm{b}} \pi \pi^{*}\right)$ to the ${ }^{1}\left(\mathrm{n}_{\mathrm{A}} \pi^{*}\right)$ state is also here envisaged based on the energies at the 2NN ground state minimum (see Table 2): the two states are separated by $0.20 \mathrm{eV}$, for which it is reasonable to expect that a stabilization of the ${ }^{1}\left(\mathrm{~L}_{b} \pi \pi^{*}\right)$ state will lead to a further reduction of such a gap and to a consequently high probability of decay to the ${ }^{1}\left(\mathrm{n}_{\mathrm{A}} \pi^{*}\right)$ state. It should be noted that the measurements were conducted in acetonitrile, and in their TDDFT computations such an environment was simulated using a PCMlike model. It can occur that in the solvent the ordering of the states may differ with respect to the gas-phase, and that a $\pi \pi^{*}$ instead of the $n \pi^{*}$ state becomes the $\mathrm{S}_{1}$ root. On the basis of a simple analysis based on the value of the dipole moments (see Table S2) an inversion of the ${ }^{1}\left(\mathrm{~L}_{b} \pi \pi^{*}\right)$ and ${ }^{1}\left(\mathrm{n}_{\mathrm{A}} \pi^{*}\right)$ states when passing from the gas-phase to an acetonitrile solution is not predicted, as the two states are characterized by similar values of the dipole moment. It can also be noted that the ${ }^{1}\left(\mathrm{~L}_{b} \pi \pi^{*}\right)$ state has a much smaller coupling to the triplet manifold and in particular with the ${ }^{3}\left(\pi_{\mathrm{O}} \pi^{*}\right)$ state, consequently making population of the triplet states an improbable process, in contrast to the significant triplet quantum yields recorded in the molecules. Finally, the reported $S_{1}$ oscillator strengths in the paper of Vogt and Crespo-Hernández are much closer to the ones here characterizing the ${ }^{1}\left(\mathrm{~L}_{\mathrm{a}} \pi \pi^{*}\right)$ state. Thus we assume that the TDDFT computed state is the ${ }^{1}\left(\mathrm{~L}_{\mathrm{a}} \pi \pi^{*}\right)$, an assumption supported by the partial charge-transfer character of such a root, ${ }^{38}$ the excited state energy underestimation of charge-transfer states in TDDFT, ${ }^{39}$ and the general issue related with excited state ordering when computed with such a level of theory. ${ }^{40}$

Another important point worth commenting on is the zero oscillator strength characterizing the ${ }^{1}\left(\mathrm{n}_{\mathrm{A}} \pi^{*}\right)$ state at the here obtained $2 \mathrm{NN}$ ground state minimum. Being completely planar and with $\mathrm{C}_{\mathrm{s}}$ symmetry, the transition from the ground to the ${ }^{1}\left(\mathrm{n}_{\mathrm{A}} \pi^{*}\right)$ state is forbidden. The same scenario is found in NB, whose ground state equilibrium structure is also totally planar. ${ }^{21}$ In the latter system population of the ${ }^{1}\left(\mathrm{n}_{\mathrm{A}} \pi^{*}\right)$ state is however possible due to low energy barrier along the torsional angle of the nitro group, which determines the coexistence of non-planar geometries from which ${ }^{1}\left(\mathrm{n}_{\mathrm{A}} \pi^{*}\right)$ promotions are allowed. In order to evaluate if such an explanation is also plausible for $2 \mathrm{NN}$, the properties of a structure obtained from the ${ }^{1}$ (gs) $\min 2 \mathrm{NN}$ by rotating the torsion of the nitro group by 45 degrees with respect to the plane of the naphthalene ring have been computed. At such a geometry the ground state energy has increased of only $0.03 \mathrm{eV}$ with respect to the ${ }^{1}$ (gs) min $_{2} 2 \mathrm{NN}$ minimum, while the ${ }^{1}\left(\mathrm{n}_{\mathrm{A}} \pi^{*}\right)$ oscillator strength is now equal to 0.0029 , for which it is possible to conclude that similarly to $\mathrm{NB}$, the excitation of ${ }^{1}\left(\mathrm{n}_{\mathrm{A}} \pi^{*}\right)$ in $2 \mathrm{NN}$ can take place from accessible nonplanar ground state structures.

Table 1. CASPT2 $(18,14)$ Vertical Excitation Energies of Nitronapthalenes at the Ground-State Minima $\left(\mathrm{E}_{\mathrm{VA}}, \mathrm{eV}\right)$ for the Lowest Valence Singlet and Spin Forbidden Triplet Excited States. ${ }^{\mathrm{a}}$

\begin{tabular}{|c|c|c|c|c|c|}
\hline \multicolumn{2}{|c|}{$2 \mathrm{NN}$} & \multicolumn{2}{c|}{$1 \mathrm{NN}$} & \multicolumn{2}{c|}{ 2M1NN } \\
\hline State & $\mathrm{E}_{\mathrm{VA}}(\mathrm{eV})$ & State & $\mathrm{E}_{\mathrm{VA}}(\mathrm{eV})$ & State & $\mathrm{E}_{\mathrm{VA}}(\mathrm{eV})$ \\
\hline $\mathrm{T}_{1}{ }^{3}\left(\pi \pi^{*}\right)$ & 2.66 & $\mathrm{~T}_{1}{ }^{3}\left(\pi \pi^{*}\right)$ & 2.53 & $\mathrm{~T}_{1}{ }^{3}\left(\pi \pi^{*}\right)$ & 2.63 \\
\hline $\mathrm{T}_{2}{ }^{3}\left(\mathrm{n}_{\mathrm{A}} \pi^{*}\right)$ & 3.09 & $\mathrm{~T}_{2}{ }^{3}\left(\mathrm{n}_{\mathrm{A}} \pi^{*}\right)$ & 3.10 & $\mathrm{~T}_{2}{ }^{3}\left(\mathrm{n}_{\mathrm{A}} \pi^{*}\right)$ & 3.12 \\
\hline $\mathrm{T}_{3}{ }^{3}\left(\pi_{\mathrm{O}} \pi^{*}\right)$ & 3.23 & $\mathrm{~T}_{3}{ }^{3}\left(\pi_{\mathrm{O}} \pi^{*}\right)$ & 3.32 & $\mathrm{~S}_{1}{ }^{1}\left(\mathrm{n}_{\mathrm{A}} \pi^{*}\right)$ & $3.33(0.0013)$ \\
\hline $\mathrm{S}_{1}{ }^{1}\left(\mathrm{n}_{\mathrm{A}} \pi^{*}\right)$ & $3.26(0.0000)$ & $\mathrm{S}_{1}{ }^{1}\left(\mathrm{n}_{\mathrm{A}} \pi^{*}\right)$ & $3.36(0.0041)$ & $\mathrm{T}_{3}{ }^{3}\left(\pi_{\mathrm{O}} \pi^{*}\right)$ & 3.36 \\
\hline $\mathrm{T}_{4}{ }^{3}\left(\pi \pi^{*}\right)$ & 3.43 & $\mathrm{~T}_{4}{ }^{3}\left(\pi \pi^{*}\right)$ & 3.52 & $\mathrm{~T}_{4}{ }^{3}\left(\pi \pi^{*}\right)$ & 3.56 \\
\hline $\mathrm{S}_{2}{ }^{1}\left(\mathrm{~L}_{\mathrm{b}} \pi \pi^{*}\right)$ & $3.46(0.0069)$ & $\mathrm{S}_{2}{ }^{1}\left(\mathrm{~L}_{\mathrm{b}} \pi \pi^{*}\right)$ & $3.70(0.0021)$ & $\mathrm{S}_{2}{ }^{1}\left(\mathrm{~L}_{\mathrm{b}} \pi \pi^{*}\right)$ & $3.61(0.0160)$ \\
\hline $\mathrm{T}_{5}{ }^{3}\left(\mathrm{n}_{\mathrm{B}} \pi^{*}\right)$ & 3.70 & $\mathrm{~T}_{5}{ }^{3}\left(\mathrm{n}_{\mathrm{B}} \pi^{*}\right)$ & 3.73 & $\mathrm{~T}_{5}{ }^{3}\left(\mathrm{n}_{\mathrm{B}} \pi^{*}\right)$ & 3.72 \\
\hline $\mathrm{S}_{3}{ }^{1}\left(\mathrm{n}_{\mathrm{B}} \pi^{*}\right)$ & $3.79\left(10^{-5}\right)$ & $\mathrm{S}_{3}{ }^{1}\left(\mathrm{n}_{\mathrm{B}} \pi^{*}\right)$ & $3.79(0.0005)$ & $\mathrm{S}_{3}{ }^{1}\left(\mathrm{n}_{\mathrm{B}} \pi^{*}\right)$ & $3.89\left(10^{-5}\right)$ \\
\hline $\mathrm{S}_{4}{ }^{1}\left(\mathrm{~L}_{\mathrm{a}} \pi \pi^{*}\right)$ & $4.30(0.1764)$ & $\mathrm{S}_{4}{ }^{1}\left(\mathrm{~L}_{\mathrm{a}} \pi \pi^{*}\right)$ & $4.05(0.1057)$ & $\mathrm{S}_{4}{ }^{1}\left(\mathrm{~L}_{\mathrm{a}} \pi \pi^{*}\right)$ & $4.12(0.1159)$ \\
\hline
\end{tabular}

${ }^{\mathrm{a}}$ The computed oscillator strengths for the singlet-singlet transitions are reported in parentheses. 
One of the main focuses of the present work is the explanation of the recorded trend for the triplet quantum yield. Since the latter has been measured exciting the systems into the $S_{1}$ state, after having characterized the excited states at the Franck-Condon regions, as described above, the following exploration of the potential surfaces was made computing two singlet and two triplet states. The corresponding Franck-Condon energies are reported in Table 2. Compared to the 5-roots results, a blue shift in the ${ }^{1}\left(\mathrm{n}_{\mathrm{A}} \pi^{*}\right)$ state is observed, leading to the lost of degeneracy with the triplet ${ }^{3}\left(\pi_{\mathrm{O}} \pi^{*}\right)$ state, whose energetic position appears to be less affected by the number of computed roots. From the three ground state minima, the relaxation experienced by the ${ }^{1}\left(\mathrm{n}_{\mathrm{A}} \pi^{*}\right)$ state has been evaluated computing the corresponding excited-state minima, performing CASSCF $(18,14)$ optimizations. Attempts to optimize the ${ }^{1}\left(\mathrm{n}_{\mathrm{A}} \pi^{*}\right)$ state of the three molecules at the CASPT2 level were unsuccessful with the energy continually fluctuating during the procedure. The so-obtained equilibrium geometries, hereafter indicated as ${ }^{1}\left(\mathrm{n}_{\mathrm{A}} \pi^{*}\right)_{\min }$, are shown in Figure 3 , while the energies of the low-lying singlet and triplet states are reported in Table 2. To verify that true CASSCF minima have been obtained, the corresponding CASSCF frequencies have been calculated. No imaginary frequencies were present. In the three minima the ${ }^{1}\left(\mathrm{n}_{\mathrm{A}} \pi^{*}\right)$ state is almost degenerate in energy with the triplet ${ }^{3}\left(\pi_{\mathrm{O}} \pi^{*}\right)$ state (energy difference equal to $0.07,0.05$, and $0.05 \mathrm{eV}$ for $2 \mathrm{NN}$, $1 \mathrm{NN}$, and $2 \mathrm{M} 1 \mathrm{NN}$, respectively) and again a large SOC characterizes the two roots $(67.36,65.00$, and $65.99 \mathrm{~cm}^{-1}$, respectively). Regarding the ${ }^{1}\left(\mathrm{n}_{\mathrm{A}} \pi^{*}\right)_{\min }$ geometries, all three minima are non-planar structures in which the nitro group displays a partial pyrimidalization.

Table 2. CASPT2 $(18,14)$ Energies $(\mathrm{eV})$ for the Most Relevant Singlet and Triplet States of Nitronaphthalenes at Different Critical Points. ${ }^{a}$

\begin{tabular}{|c|c|c|c|c|c|c|c|c|c|c|c|c|}
\hline & \multicolumn{4}{|c|}{$2 \mathrm{NN}$} & \multicolumn{4}{|c|}{$1 \mathrm{NN}$} & \multicolumn{4}{|c|}{ 2M1NN } \\
\hline $\begin{array}{l}\text { State } \\
\text { Geometry }\end{array}$ & gs & ${ }^{1}\left(\mathrm{n}_{\mathrm{A}} \pi^{*}\right)$ & ${ }^{3}\left(\pi_{\mathrm{O}} \pi^{*}\right)$ & $3\left(\mathrm{n}_{\mathrm{A}} \pi^{*}\right)$ & gs & ${ }^{1}\left(\mathrm{n}_{\mathrm{A}} \pi^{*}\right)$ & ${ }^{3}\left(\pi_{\mathrm{O}} \pi^{*}\right)$ & ${ }^{3}\left(\mathrm{n}_{\mathrm{A}} \pi^{*}\right)$ & gs & ${ }^{1}\left(\mathrm{n}_{\mathrm{A}} \pi^{*}\right)$ & ${ }^{3}\left(\pi_{0} \pi^{*}\right)$ & ${ }^{3}\left(\mathrm{n}_{\mathrm{A}} \pi^{*}\right)$ \\
\hline${ }^{1}(\mathrm{gs})_{\min }$ & 0.00 & 3.51 & 3.21 & 3.25 & 0.00 & 3.59 & 3.35 & 3.16 & 0.00 & 3.56 & 3.48 & 3.26 \\
\hline${ }^{1}\left(\mathrm{n}_{\mathrm{A}} \pi^{*}\right)_{\min }$ & 1.15 & 2.88 & 2.95 & 2.93 & 1.63 & 3.04 & 3.09 & 3.14 & 1.48 & 3.05 & 3.10 & 3.13 \\
\hline$\left({ }^{1} \mathrm{n}_{\mathrm{A}} \pi^{*} / \mathrm{gs}\right)_{\mathrm{CI}}$ & 3.13 & 3.13 & 4.49 & 3.09 & 3.16 & 3.17 & 4.55 & 3.12 & 3.04 & 3.05 & 4.64 & 3.01 \\
\hline
\end{tabular}

${ }^{a}$ All the reported values are referred to the corresponding ground state optimized geometry, ${ }^{1}(\mathrm{gs})_{\min }$.

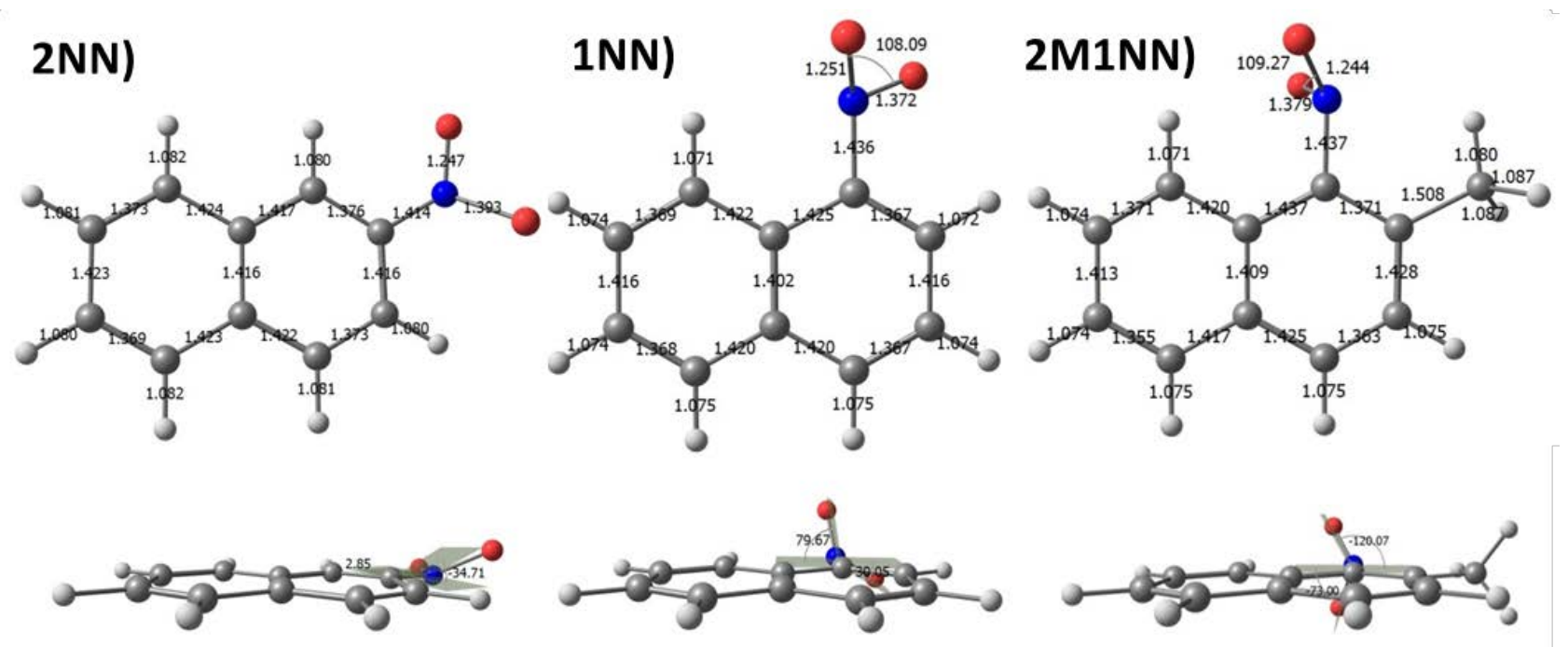

Figure 3. ${ }^{1}\left(\mathrm{n}_{\mathrm{A}} \pi^{*}\right)_{\min }$ minima of $2 \mathrm{NN}, 1 \mathrm{NN}$, and $2 \mathrm{M} 1 \mathrm{NN}$. Selected bond lengths (in $\AA$ ), angles, and dihedral angles (in degrees) are also reported. 
Based on the outcomes presented above, we can then conclude that the same static description is shared in the three molecules. The bright ${ }^{1}\left(\mathrm{n}_{\mathrm{A}} \pi^{*}\right)_{\min }$ is strongly coupled with the triplet ${ }^{3}\left(\pi_{\mathrm{O}} \pi^{*}\right)$ state, as reflected by the reported high SOC values, and in its minimum the two roots are degenerate, making the corresponding intersystem crossing process a particularly favorable outcome. This scenario was already characterized for $1 \mathrm{NN}$ and used as a rationalization for the ultrafast singlet to triplet decay experimentally recorded in the molecule. ${ }^{37,41,42}$ On the basis of the obtained static results, it is then likely that after ${ }^{1}\left(\mathrm{n}_{\mathrm{A}} \pi^{*}\right)$ absorption, the path leading to triplet state population is equally favorable in the three molecules. This consequently points out to the presence of a competing path whose presence justifies the loss in triplet quantum yield observed along the series. Such a consideration, together with the particularly low or even absent fluorescence emission in the molecules, makes plausible the presence of an accessible non-radiative decay path in competition with the singlet to triplet decay route.

In our previous article on the related system NB, a non-radiative decay from the ${ }^{1}\left(\mathrm{n}_{\mathrm{A}} \pi^{*}\right)$ to the ground state was described, involving the presence of an accessible CI between the two states. ${ }^{21}$ The latter geometry is characterized by a shortening of the CN bond, together with an enlargement of both NO distances, and a decrease in the ONO angles. In order to evaluate if such a path is also possible in the three nitronaphthalene systems, CASPT2 CI optimizations have been carried out using the projected constrained optimization method as recently implemented in the Molcas software, obtaining minimum energies crossing points (MECPs) between the ${ }^{1}\left(\mathrm{n}_{\mathrm{A}} \pi^{*}\right)$ and the ground state. MECPs are in general used as a model for the description of a CI seam and in order to evaluate the relation between other critical point and the CI region. ${ }^{43}$ Care should however be taken in the use of such a model, which is based on the assumption that MECP are the most representative point of a CI and from which internal conversion phenomena are most likely to occur. In reality, population transfer can occur at all points along a CI seam and what is in general more important is the first encountered CI point rather than the MECP, as shown for example in the photophysics of guanine. ${ }^{44}$ In the present contribution we however decided to compute MECPs as a way of characterizing the CIs, since it will provide a strategy in order to compare the CI regions in the various molecules here studied. For each system here under study a MECP has been found, hereafter denoted as $\left({ }^{1} n_{A} \pi^{*} / g s\right)_{C l}$, which displays indeed the same main geometrical deformations present in the related CI of NB (see Figure 4, Table S3, and ref. 21).

The computed MECPs can be considered to lie along the same "deformation direction" characterizing the evolution of the systems from the ground state minima towards the ${ }^{1}\left(\mathrm{n}_{\mathrm{A}} \pi^{*}\right)$ equilibrium structures: at least with respect to the nitro group, the MECPs geometries display the same major deformations seen in the ${ }^{1}\left(\mathrm{n}_{\mathrm{A}} \pi^{*}\right)$ minima. These are, as in the case of NB, a decrease in the CN distance and ONO angle, together with an enlargement of the NO bonds (see Figures 2-4, and Table S3).

Comparing the energies of the low-lying singlet and triplet states at the computed crossing point to the energies of the ground-state and ${ }^{1}\left(n_{A} \pi^{*}\right)$ minima (see Table 2), the following observations can be drawn. First, each MECP is energetically below the corresponding ${ }^{1}\left(\mathrm{n}_{\mathrm{A}} \pi^{*}\right)$ vertical excitation energy at the ground state equilibrium structure, specifically $0.38,0.42$, and $0.51 \mathrm{eV}$ lower in energy for $2 \mathrm{NN}, 1 \mathrm{NN}$, and $2 \mathrm{M} 1 \mathrm{NN}$, respectively. This in turn means that after excitation each of the three molecules should have enough energy to reach the corresponding CI. Second, the energy difference between the ${ }^{1}\left(\mathrm{n}_{\mathrm{A}} \pi^{*}\right)$ minimum and the MECP increases along the series: $0.25 \mathrm{eV}$ for $2 \mathrm{NN}, 0.13 \mathrm{eV}$ for $1 \mathrm{NN}$, and $0.00 \mathrm{eV}$ for $2 \mathrm{M} 1 \mathrm{NN}$. The trend in the energy differences shows that the accessibility of the CI region becomes more and more favorable along the series of molecules, following the same trend as the experimental value of the triplet quantum yield. 


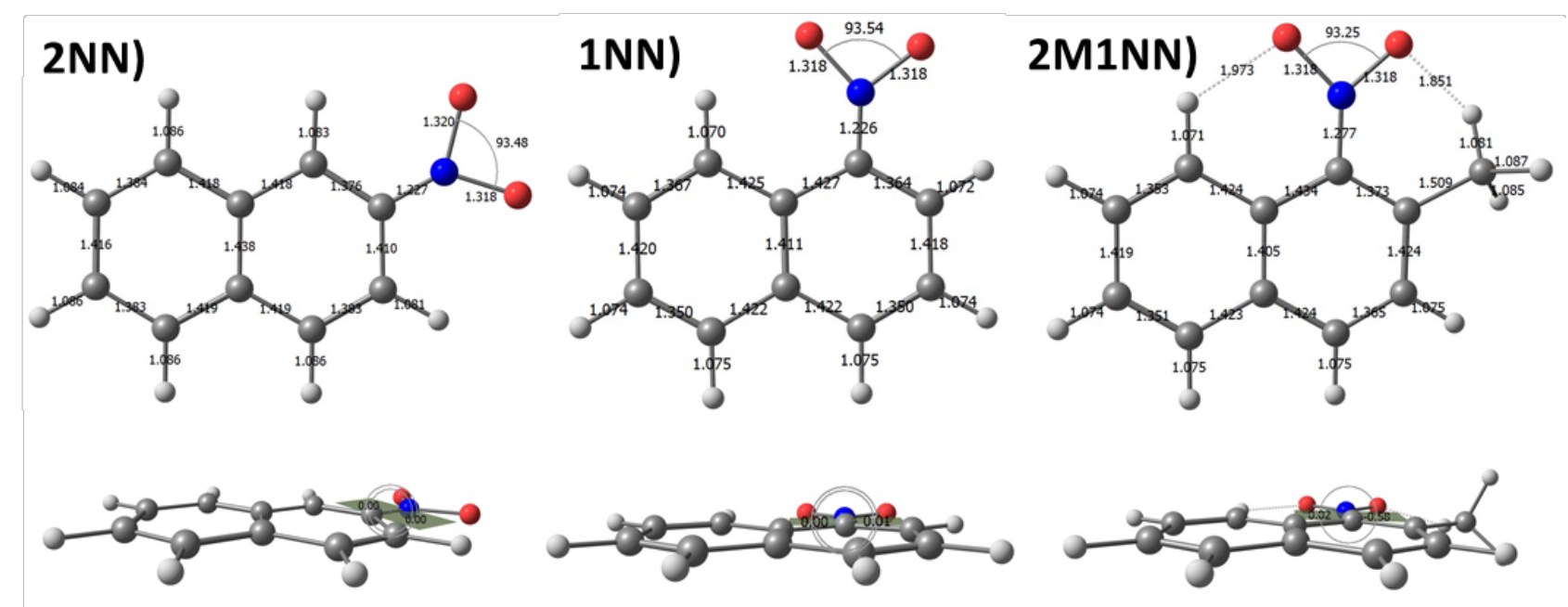

Figure 4. $\left({ }^{1} \mathrm{n}_{\mathrm{A}} \pi^{*} / \mathrm{gs}\right)_{\mathrm{CI}} \mathrm{MECP}$ of $2 \mathrm{NN}, 1 \mathrm{NN}$, and $2 \mathrm{M} 1 \mathrm{NN}$. Selected bond lengths (in $\AA$ ), angles, and dihedral angles (in degrees) are also reported.

In order to better characterize the potential energy hypersurfaces between the ${ }^{1}\left(\mathrm{n}_{\mathrm{A}} \pi^{*}\right)$ minima and the related MECP, LIIC computations between the two critical points have been performed. The computed CASPT2 $(18,14)$ energies along these are presented in Table 3. As shown in Table 3, the ${ }^{1}\left(\mathrm{n}_{\mathrm{A}} \pi^{*}\right)$ energy initially decreases along the LIIC paths, despite the fact that the starting points were the ${ }^{1}\left(\mathrm{n}_{\mathrm{A}} \pi^{*}\right)$ minima. The behavior is probable due to the fact that the minima were obtained at the CASSCF $(18,14)$ level, while the final energies are evaluated performing CASPT2 $(18,14)$ calculations. Evaluating the energy barrier as the difference between the lowest point along the LIIC path and the MECP, values were obtained that again follow the same trend as the one shown for the triplet quantum yields: $0.47,0.42$, and $0.19 \mathrm{eV}$ for $2 \mathrm{NN}, 1 \mathrm{NN}$, and $2 \mathrm{M} 1 \mathrm{NN}$, respectively.

Based on the results, we can formulate the following model that rationalizes the trend for the values of the triplet quantum yield in the molecules $2 \mathrm{NN}, 1 \mathrm{NN}$, and $2 \mathrm{M} 1 \mathrm{NN}$. After excitation into the $\mathrm{S}_{1}$ ${ }^{1}\left(\mathrm{n}_{\mathrm{A}} \pi^{*}\right)$ state, the latter evolves in the three systems toward a minimum structure characterized by a strong coupling with the triplet ${ }^{3}\left(\pi_{\mathrm{O}} \pi^{*}\right)$ state (large SOC and almost degenerate energy). This consequently constitutes a particularly favorable decay path leading into the triplet manifold. In the three molecules a non-radiative internal conversion decay path coexists along with the decay route into the triplet state, mediated by the presence of a CI between the ${ }^{1}\left(\mathrm{n}_{\mathrm{A}} \pi^{*}\right)$ and the ground state. While the decay path leading to triplet state population is equally favorable in the systems, the nonradiative re-population of the ground state appear to be more and more favorable along the series of molecules, as reflected by the decreasing value of the energy barrier needed in order to reach the corresponding MECP from the related ${ }^{1}\left(\mathrm{n}_{\mathrm{A}} \pi^{*}\right)$ minimum. The importance that the non-radiative decay path acquires along the series makes it competitive with the triplet route, consequently determining the lose of triplet quantum yield experimentally registered. The decay paths are summarised in Figure 5.

The computed energies barriers are not very high, and in addition it needs to be taken into account that they have been obtained from LIIC paths, meaning that the reported values constitute upper bounds of the true energies barriers. This fact, together with the favorable geometrical disposition of the excited minima and MECPs (i.e. being placed along the same deformation direction for the nitro group) means that the non-radiative internal conversion decay should be relatively efficient. Nevertheless, most of the excited-state population, except for $2 \mathrm{M} 1 \mathrm{NN}$, decays into the triplet manifold, indicating that despite the presence of an accessible non-radiative decay, the singlettriplet coupling is strong enough to make the intersystem crossing process the most favorable relaxation route. It should be noticed that energy barriers of around $0.2 \mathrm{eV}$ have been associated to 
excited state lifetimes of the order of the picosecond in DNA related systems; ${ }^{45}$ considering that nitroaromatics systems can have ultrafast decay into the triplet manifold (as in $1 \mathrm{NN}$, whose triplet states is populated in $100 \mathrm{fs}$ after $\mathrm{S}_{1}$ absorption) it is then justifiable than the triplet route remains the main decay route in the nitroaromatics systems here studied.

In order to further validate the present static model, the latter has also been applied to the related system NB, in which the presence of a $\left({ }^{1} n_{A} \pi^{*} / g s\right)_{C I}$ CI was originally described. From an optimized minimum energy crossing point obtained starting from the NB CASPT2 $(14,11)$ CI, the corresponding LIIC path between this crossing region and the previously published CASSCF $(14,11)^{1}\left(n_{A} \pi^{*}\right)$ minimum has been computed. ${ }^{21}$ A similar scenario to the one described for the nitronaphthalene systems has resulted, obtaining an energy difference between the minimum and the MECP of $0.35 \mathrm{eV}$, and an energy difference between the lowest LIIC point and the MECP of $0.48 \mathrm{eV}$ (see Table 3). Performing picosecond time-resolved transient grating measurements in ethanol after excitation into the NB $\mathrm{S}_{1}$ state, Terazima and co-workers determined that the triplet quantum yield of NB is greater than $0.8{ }^{46,47}$ Such quantities match with the proposed model: a 0.36 (0.48) eV barrier match with a triplet quantum yield greater that the one for $1 \mathrm{NN}$ and $2 \mathrm{M} 1 \mathrm{NN}$, having respectively a $0.13(0.42) \mathrm{eV}$ and $0.00(0.19) \mathrm{eV}$ energy barrier, and of the order of the triplet quantum yield characterizing the 2NN molecule, for which a barrier of $0.25(0.47)$ eV have been obtained. As NB is the system for which the highest barrier has been computed, we would expect that the molecule displays the larger triplet quantum yield, a hypothesis that is plausible with the experimental determination of a value greater than 0.8 .

It is interesting to notice that the system characterized by the higher energy gaps between the ${ }^{1}\left(\mathrm{n}_{\mathrm{A}} \pi^{*}\right)$ minimum and the corresponding MECP, i.e. NB , is also the system displaying the smallest geometrical differences between such two critical points. In NB both the minimum and the MECPs are totally planar, and the same main deformations are present. In the $2 \mathrm{NN}, 1 \mathrm{NN}$, and $2 \mathrm{M} 1 \mathrm{NN}$ molecules, the $\left({ }^{1} n_{A} \pi^{*} / g s\right)_{C I}$ MECPs are again planar structures, but instead in the corresponding ${ }^{1}\left(\mathrm{n}_{\mathrm{A}} \pi^{*}\right)$ minima the nitro group is no longer coplanar with the naphthalene ring, and in both cases it has lost its own planar conformation displaying a certain degree of pyrimidalization. Despite the larger geometrical deformations required in $2 \mathrm{NN}, 1 \mathrm{NN}$, and $2 \mathrm{M} 1 \mathrm{NN}$ than in $\mathrm{NB}$ for evolving form the ${ }^{1}\left(\mathrm{n}_{\mathrm{A}} \pi^{*}\right)$ minimum to the $\left({ }^{1} \mathrm{n}_{\mathrm{A}} \pi^{*} / \mathrm{gs}\right)_{\mathrm{CI}}$ MECP, the former systems are, as reported above, characterized by a lower energy barrier than NB.

As a final consideration, and since the present model is also based on the energy of the ${ }^{1}\left(\mathrm{n}_{\mathrm{A}} \pi^{*}\right)$ minimum, the 2NN CASSCF $(18,14)$ equilibrium structure has been re-optimized starting from the previously described geometry obtained from the 2NN ground state minimum in which the nitro group has been placed at 45 degrees with respect to the plane of the rings. From such a geometry, characterized by a significant value of the oscillator strength for the electronic promotion into the ${ }^{1}\left(\mathrm{n}_{\mathrm{A}} \pi^{*}\right)$ state, the ${ }^{1}\left(\mathrm{n}_{\mathrm{A}} \pi^{*}\right) \operatorname{CASSCF}(18,14)$ optimization has ended in the same region as described above, consequently validating the conclusion drawn from such a minimum.

Table 3. CASPT2 $(18,14)$ Computed Energies $(\mathrm{eV})$ for the ${ }^{1}\left(\mathrm{n}_{\mathrm{A}} \pi^{*}\right)$ States of Nitroaromatics Along the Computed LIIC Paths between ${ }^{1}\left(\mathrm{n}_{\mathrm{A}} \pi^{*}\right)_{\min }$ Minima and $\left({ }^{1} \mathrm{n}_{\mathrm{A}} \pi^{*} / \mathrm{gs}\right)_{\mathrm{CI}}$ MECPs.

\begin{tabular}{|c|c|c|c|c|c|c|c|c|c|c|c|}
\hline & \multicolumn{10}{|c|}{ Geometry } \\
\hline Molecule & ${ }^{1}\left(\mathrm{n}_{\mathrm{A}} \pi^{*}\right)_{\min }$ & LIICg1 & LIICg2 & LIICg3 & LIICg4 & LIICg5 & LIICg6 & LIICg7 & LIICg8 & $\begin{array}{c}\left(^{1}\right. \\
\left.\mathrm{n}_{\mathrm{A}} \pi^{*} / \mathrm{gs}\right)_{\mathrm{CI}}\end{array}$ & $\Delta \mathrm{E}^{\mathrm{a}}$ \\
\hline $2 \mathrm{NN}$ & $\mathbf{2 . 8 8}$ & 2.75 & 2.77 & 2.73 & 2.69 & $\mathbf{2 . 6 6}$ & 2.67 & 2.73 & 2.88 & $\mathbf{3 . 1 3}$ & 0.47 \\
\hline $1 \mathrm{NN}$ & $\mathbf{3 . 0 4}$ & 2.96 & 2.96 & 2.83 & 2.78 & $\mathbf{2 . 7 5}$ & 2.75 & 2.89 & 3.03 & $\mathbf{3 . 1 7}$ & 0.42 \\
\hline $2 \mathrm{M} 1 \mathrm{NN}$ & $\mathbf{3 . 0 5}$ & 3.01 & 2.91 & 2.91 & 2.90 & 2.88 & $\mathbf{2 . 8 6}$ & 2.86 & 3.00 & $\mathbf{3 . 0 5}$ & 0.19 \\
\hline NB & $\mathbf{2 . 8 5}$ & 2.79 & 2.75 & 2.73 & $\mathbf{2 . 7 2}$ & 2.74 & 2.79 & 2.88 & 3.01 & $\mathbf{3 . 2 0}$ & 0.48 \\
\hline
\end{tabular}

${ }^{\mathrm{a}}$ Energy difference $(\mathrm{eV})$ between the MECP and the lowest computed point along the LIIC. 


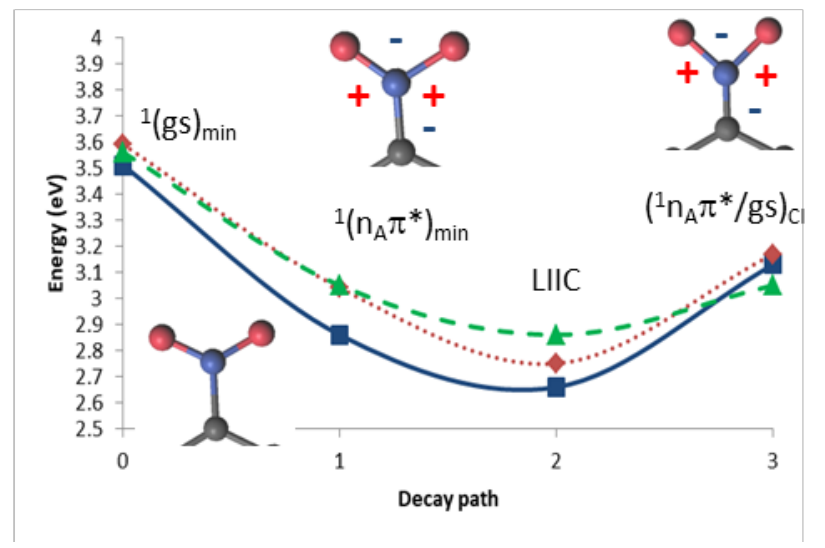

Figure 5. Schematic representation of the CASPT2 $(18,14)$ PEHs of the ${ }^{1}\left(\mathrm{n}_{\mathrm{A}} \pi^{*}\right)$ state for the $2 \mathrm{NN}$, $1 \mathrm{NN}$, and $2 \mathrm{M} 1 \mathrm{NN}$ molecules along the characterized critical points. Solid blue, dotted red, and dashed green lines correspond to the ${ }^{1}\left(\mathrm{n}_{\mathrm{A}} \pi^{*}\right)$ state for the $2 \mathrm{NN}, 1 \mathrm{NN}$, and $2 \mathrm{M} 1 \mathrm{NN}$ systems, respectively. The main geometrical deformations of the nitro group are reported employing red plus ${ }^{+}+$and minus blue symbols, indicating an increase and decrease, respectively, with respect to the $\mathrm{S}_{0}$ minima (see Table S3)

\section{Conclusions}

On the basis of high-level ab initio CASPT2//CASSCF and CASPT2 computations, a model rationalizing the experimental trend of the triplet quantum yield for the molecules $2 \mathrm{NN}, 1 \mathrm{NN}$, and $2 \mathrm{M} 1 \mathrm{NN}\left(0.93 \pm 0.15,0.64 \pm 0.12\right.$, and $0.33 \pm 0.05$, respectively) after excitation into the $\mathrm{S}_{1}$ state has been proposed. The remarkable decrease in the triplet quantum yield observed along the series of molecules is shown here not to be caused by an increasing difficulty in reaching the singlettriplet crossing region leading to the triplet manifold, but to be attributable the an increase in the possibility of instead decaying along a competitive non-radiative internal conversion path mediated by an accessible non-previously reported conical intersection between the $\mathrm{S}_{1}$ and the ground-state. The $S_{1}$ state in the three molecules have been here characterized to have the same $n \pi^{*}$ nature, in agreement with previous CASPT2//CASSCF computation ${ }^{37,41}$ but in dissonance with previous TDDFT computations. ${ }^{18,19}$

The $\mathrm{S}_{1}$ state evolves in the three systems towards an equilibrium structure in which it is degenerate and strongly coupled with a triplet state, which can consequently be easily populated. This decay is common to the three molecules, for which we conclude that the path leading to the intersystem crossing process is equally favorable in all the here studied molecules. From this $S_{1}$ minimum, the systems can also further evolve towards the mentioned $S_{1} / S_{0}$ conical intersection, and consequently decay non-radiatively. The probability to evolve along this non-radiative path appears to be systemdependent, determined by the computed energy barriers to reach the conical intersection from the $S_{1}$ minimum in the three systems. The values of these barriers follow the same trend as the experimental values of the triplet quantum yields: larger for the systems characterized by a larger triplet quantum yield.

In order to compare the three CI regions in the three systems, CASPT2 minimum-energy crossing point for each CI have been obtained. The derived model has also been applied to the related system $\mathrm{NB}$, and the results obtained match with the experimental value of the triplet quantum yield for that molecule. 
The present contribution thus sheds light onto the remarkably different photophysical behavior that the nitronaphthalene molecules display, despite their similar chemical structures. This in turn constitutes a nice example of how presumably small chemical modifications (such as the functional group position and methylation) can have dramatic effects in the system behavior, and should be taken into consideration when comparing presumably similar systems, as for example thymine and uracil, and cytosine and 5methyl-cytosine, two pairs of molecules differentiated by a methyl group but characterized by different photoresponses.

\section{Author information \\ Corresponding Author \\ *E-mail: angelo.giussani@uv.es.}

\section{ORCID}

Angelo Giussani: 0000-0002-9452-7641

Notes

The authors declare no competing financial interest.

\section{Acknowledgements}

This project has received funding from the European Union's Horizon 2020 research and innovation programme under the Marie Sklodowska-Curie Grant Agreement No. 658173. A.G. also thanks Spanish MICINN (CTQ2017-87054-C2-2-P) Unidad de Excelencia María de Maetzu MDM-20150538), and Generalitat Valenciana (PROMETEO/2016/135)

\section{REFERENCES}

1 T. Handa, T. Yamauchi, M. Ohnishi, Y. Hisematsu and T. Ishii, Detection and average content levels of carcinogenic and mutagenic compounds from the particulates on diesel and gasoline engine mufflers, Environ. Int., 1983, 9, 335.

2 L. Möller, I. Lax and L. C. Eriksson, Nitrated Polycyclic Aromatic Hydrocarbons: A Risk Assessment for the Urban Citizen, Environ. Heal. Perspect., 1993, 101, 309.

3 X. Xu, J. Nachtman, S. Rappaport and E. Wei, Identification of 2-Nitrofluorene in diesel exhaust particulates, J. Appl. Toxicol., 1981, 1, 196.

4 Y. I. Y. Wang, S. M. Rappaport, R. F. Sawyer, R. E. Talcott and E. T. Wei, Direct-acting mutagens in automobile exhaust, Cancer Lett., 1987, 5, 39.

5 J. A. Miller, R. B. Sandin, E. C. Miller and H. P. Rush, The Carcinogenicity of Compounds Related to 2-Acetylaminofluorene, Cancer Res., 1955, 15, 188.

6 A. K. Sikder, G. Maddala, J. P. Agrawal and H. Singh, Important aspects of behaviour of organic energetic compounds: a review, J. Hazard. Mater., 2001, 84, 1-26.

7 H. Nakagawa, K. Hishikawa, K. Eto, N. Ieda, T. Namikawa, K. Kamada, T. Suzuki, N. Miyata and J. I. Nabekura, Fine spatiotemporal control of nitric oxide release by infrared pulse-laser irradiation of a photolabile donor, ACS Chem. Biol., 2013, 8, 2493-2500.

8 O. L. Chapman, D. C. Heckert, J. W. Reasoner and S. P. Thackaberry, Photochemical Studies on 9-Nitroanthracene1, J. Am. Chem. Soc., 1966, 88, 5550-5554. 
K. Fukuhara, M. Kurihara and N. Miyata, Photochemical Generation of Nitric Oxide from 6Nitrobenzo[a]pyrene, J. Am. Chem. Soc., 2001, 123, 8662-8666.

10 G. Y. Gerasimov, Photochemical Degradation of Polycyclic Aromatic and Nitroaromatic Hydrocarbons in the Urban Atmosphere, High Energy Chem., 2004, 38, 161-166.

11 P. T. Phousongphouang and J. Arey, Rate constants for the photolysis of the nitronaphthalenes and methylnitronaphthalenes, J. Photochem. Photobiol. A Chem., 2003, 157, 301-309.

12 D. B. Galloway, J. A. Bartz, L. G. Huey and F. F. Crim, Pathways and Kinetic Energy Disposal in the Photodissociation of Nitrobenzene, J. Chem. Phys., 1993, 98, 2107.

13 M. L. Hause, N. Herath, R. Zhu, M. C. Lin and A. G. Suits, Roaming-Mediated Isomerization in the Photodissociation of Nitrobenzene, Nat. Chem., 2011, 3, 932.

14 R. Morales-Cueto, M. Esquivelzeta-Rabell, J. Saucedo-Zugazagoitia and J. Peon, Singlet Excited-State Dynamics of Nitropolycyclic Aromatic Hydrocarbons: Direct Measurements by Femtosecond Fluorescence Up-Conversion, J. Phys. Chem. A, 2007, 111, 552-557.

15 S. J. Zugazagoitia, C. X. Almora-Díaz and J. Peon, Ultrafast Intersystem Crossing in 1Nitronaphthalene. An Experimental and Computational Study, J. Phys. Chem. A, 2008, 112, 358.

16 J. S. Zugazagoitia, E. Collado-fregoso, E. F. Plaza-medina and J. Peon, Relaxation in the Triplet Manifold of 1-Nitronaphthalene Observed by Transient Absorption Spectroscopy, $J$. Phys. Chem. A, 2009, 113, 805-810.

17 R. A. Vogt, C. Reichardt and C. E. Crespo-herna, Excited-State Dynamics in NitroNaphthalene Derivatives: Intersystem Crossing to the Triplet Manifold in Hundreds of Femtoseconds, J. Phys. Chem. A, 2013, 117, 6580-6588.

18 J. P. Zobel, J. J. Nogueira and L. González, Mechanism of Ultrafast Intersystem Crossing in 2-Nitronaphthalene, Chem. - A Eur. J., 2018, 24, 5379-5387.

19 R. A. Vogt and C. E. Crespo-Hernandez, Conformational Control in the Population of the Triplet State and Photoreactivity of Nitronaphthalene Derivatives, J. Phys. Chem. A, 2013, 117, 14100-14108.

20 C. E. Crespo-Hernández, R. A. Vogt and B. Sealey, On the Primary Reaction Pathways in the Photochemistry of Nitro-Polycyclic Aromatic Hydrocarbons, Mod. Chem. Appl., 2013, 1, 17.

21 A. Giussani and G. A. Worth, Insights into the Complex Photophysics and Photochemistry of the Simplest Nitroaromatic Compound: A CASPT2//CASSCF Study on Nitrobenzene, J. Chem. Theory Comput., 2017, 13, 2777-2788.

22 K. Andersson, P.-A. Malmqvist and B. O. Roos, Second-order perturbation theory with a complete active space self-consistent field reference function, J. Chem. Phys., 1992, 96, 1218. 
23 B. O. Roos, K. Andersson, M. P. Fülscher, P.-Å. Malmqvist, L. Serrano-Andrés, K. Pierloot and M. Merchán, Multiconfigurational Perturbation Theory: Applications in Electronic Spectroscopy, Adv. Chem. Phys., 1996, 93, 219.

24 L. Serrano-Andrés, M. Merchán, P. v. R. Schleyer, P. R. Schreiner, H. F. Schaefer, W. L. Jorgensen, W. Thiel and R. C. Glen, Encyclopedia of Computational Chemistry, 2004.

25 F. Aquilante, J. Autschbach, R. Carlson, L. Chibotaru, M. G. Delcey, L. De Vico, I. Fernández Galvan, N. Ferré, L. M. Frutos, L. Gagliardi, M. Garavelli, A. Giussani, C. Hoyer, G. Li Manni, H. Lischka, D. Ma, P. A. Malmqvist, T. Müller, A. Nenov, M. Olivucci, T. B. Pedersen, D. Peng, F. Plasser, B. Pritchard, M. Reiher, I. Rivalta, I. Schapiro, J. SegarraMartí, M. Stenrup, D. G. Truhlar, L. Ungur, A. Valentini, S. Vancoillie, V. Veryazov, V. Vysotskiy, O. Weingart, F. Zapata and R. Lindh, MOLCAS 8: New Capabilities for Multiconfigurational Quantum Chemical Calculations across the Periodic Table, J. Comput. Chem., 2016, 37, 506.

26 P.-O. Widmark, P.-Å. Malmqvist and B. O. Roos, Density Matrix Averaged Atomic Natural Orbital (ANO) Basis Sets for Correlated Molecular Wave Functions, Theor. Chem. Acc., 1990, 77, 291.

27 K. Pierloot, B. Dumez, P.-O. Widmark and B. O. Roos, Density Matrix Averaged Atomic Natural Orbital (ANO) Basis Sets for Correlated Molecular Wave Functions, Theor. Chim. Acta, 1995, 90, 87.

28 M. Merchán, L. Serrano-Andrés and M. Olivucci, Ab initio methods for excited states, In Computational Photochemistry, ed. M. Olivucci, Elsevier, Amsterdam, 1st edn., 2005, vol. 16, ch. 1, pp. 35-91

29 A. Giussani, R. Pou-Amérigo, L. Serrano-Andrés, A. Freire-Corbacho, C. Martínez-García, M. I. Fernández P, M. Sarakha, M. Canle L and J. A. Santaballa, Combined theoretical and experimental study of the photophysics of asulam., J. Phys. Chem. A, 2013, 117, 2125-37.

30 A. Giussani, L. Serrano-Andrés, M. Merchán, D. Roca-Sanjuán and M. Garavelli, Photoinduced Formation Mechanism of the Thymine-Thymine (6-4) Adduct, J. Phys. Chem. B, 2013, 117, 1999.

31 I. F. Galván, M. G. Delcey, T. B. Pedersen, F. Aquilante and R. Lindh, Analytical StateAverage Complete-Active-Space Self-Consistent Field Nonadiabatic Coupling Vectors: Implementation with Density-Fitted Two-Electron Integrals and Application to Conical Intersections, J. Chem. Theory Comput., 2016, 12, 3636-3653.

32 A. Giussani, M. Merchán, J. P. Gobbo and A. C. Borin, Relaxation Mechanisms of 5Azacytosine, J. Chem. Theory Comput., 2014, 10, 3915.

33 M. Merchán, L. Serrano-Andrés, M. A. Robb and L. Blancafort, Triplet-State Formation along the Ultrafast Decay of Excited Singlet Cytosine, J. Am. Chem. Soc., 2005, 127, 1820.

34 T. B. Pedersen, R. Lindh and F. Aquilante, Density fitting with auxiliary basis sets from Cholesky decompositions, Theor. Chem. Acc., 2009, 124, 1. 
35 A. Domenicano, G. Schultz, I. Hargittai, M. Colapietro, G. Portalone, P. George and C. W. Bock, Molecular structure of nitrobenzene in the planar and orthogonal conformations - A concerted study by electron diffraction, X-ray crystallography, and molecular orbital calculations, Struct. Chem., 1990, 1, 107-122.

36 J.-M. Mewes, V. Jovanović, C. M. Marian and A. Dreuw, On the Molecular Mechanism of Non-Radiative Decay of Nitrobenzene and the Unforeseen Challenges this Simple Molecule Holds for Electronic Structure Theory, Phys. Chem. Chem. Phys., 2014, 16, 12393.

37 A. Giussani, Toward the Understanding of the Photophysics and Photochemistry of 1Nitronaphthalene under Solar Radiation: The First Theoretical Evidence of a Photodegradation Intramolecular Rearrangement Mechanism Involving the Triplet States, J. Chem. Theory Comput., 2014, 10, 3987-3995.

38 J. P. Zobel, J. J. Nogueira and L. González, Quenching of Charge Transfer in Nitrobenzene Induced by Vibrational Motion, J. Phys. Chem. Lett., 2015, 6, 3006.

39 A. Dreuw, J. L. Weisman and M. Head-Gordon, Long-range charge-transfer excited states in time-dependent density functional theory require non-local exchange, J. Chem. Phys., 2003, 119, 2943-2946.

40 S. Arulmozhiraja and M. L. Coote, $1 \mathrm{La}$ and $1 \mathrm{Lb}$ States of Indole and Azaindole: Is Density Functional Theory Inadequate ?, J. Chem. Theory Comput., 2012, 8, 575-584.

41 Y. Orozco-Gonzalez, K. Coutinho, J. Peon and S. Canuto, Theoretical Study of the Absorption and Nonradiative Deactivation of 1-Nitronaphthalene in the Low-Lying Singlet and Triplet Excited States Including Methanol and Ethanol Solvent Effects, J. Chem. Phys., 2012, 137, 54307.

42 M. Yang, T. Zhang, J. Xue and X. Zheng, Ab Initio Study of Decay Dynamics of 1Nitronaphthalene Initiated from the $\mathrm{S} 2(\pi \pi *+\mathrm{nNO}$ ) State, J. Phys. Chem. A, 2018, 122, 2732-2738.

43 J. J. Serrano-Andrés, Luis ; Serrano-Pérez, Calculation of excited states: molecular photophysics and photochemistry on display, in Handbook of Computational Chemistry, ed. J. Leszczynski, Springer-Verlang, 2012, ch. 14, pp. 483-560.

44 L. Serrano-Andrés, M. Merchán and A. C. Borin, A Three-State Model for the Photophysics of Guanine, J. Am. Chem. Soc., 2008, 130, 2473-2484.

45 Q. Li, A. Giussani, J. Segarra-Martí, A. Nenov, I. Rivalta, A. A. Voityuk, S. Mukamel, D. Roca-Sanjuán, M. Garavelli and L. Blancafort, Multiple Decay Mechanisms and 2D-UV Spectroscopic Fingerprints of Singlet Excited Solvated Adenine-Uracil Monophosphate, Chem. - Eur. J., 2016, 22, 7497.

46 M. Takezaki, N. Hirota and M. Terazima, Nonradiative Relaxation Processes and Electronically Excited States of Nitrobenzene Studied by Picosecond Time-Resolved Transient Grating Method, J. Phys. Chem. A, 1997, 101, 3443.

47 M. Takezaki, N. Hirota and M. Terazima, Relaxation of Nitrobenzene from the Excited Singlet State, J. Chem. Phys., 1998, 108, 4685. 